\title{
Inspiratory pressure support prolongs exercise induced lactataemia in severe COPD
} Michael I Polkey, Peter Hawkins, Dimitris Kyroussis, Sheric G Ellum, Roy Sherwood,
John Moxham
Methods

Background-A physiological benefit
from pulmonary rehabilitation in chronic
obstructive pulmonary disease (COPD) is
more probable if exercise is performed
above the lactate threshold. This study
was undertaken to investigate whether it
was possible to extend the lactataemia of
exercise using non-invasive inspiratory
pressure support (IPS). Methods-Plasma lactate levels were measured in eight men with severe COPD who performed two treadmill walks at an identical constant work rate to a condition of severe dyspnoea; the second walk was supported by IPS.

Results-Mean plasma lactate levels before the free and IPS assisted walks were $1.65 \mathrm{mmol} / 1$ and $1.53 \mathrm{mmol} / 1$, respectively $(\mathbf{p}=\mathrm{NS})$. Lactate levels increased during both walks to $2.96 \mathrm{mmol} / 1$ and $2.42 \mathrm{mmol} /$ 1 , respectively ( $p=0.01$ for each) but the duration of the IPS assisted walk was significantly greater than the free walk (13.6 minutes versus 5.5 minutes, $p=0.01$ ).

Conclusions-Patients with severe COPD can sustain exercise induced lactataemia for longer if assisted with IPS. This technique may prove to be a useful adjunct in pulmonary rehabilitation.

(Thorax 2000;55:547-549)

Keywords: chronic obstructive pulmonary disease; exercise; lactataemia; inspiratory pressure support

Patients with moderate chronic obstructive pulmonary disease (COPD) derive a greater physiological training benefit if exercise is performed at a work rate which exceeds their anaerobic threshold. ${ }^{1}$ One marker of anaerobic muscle metabolism used in clinical studies is the presence of an increased plasma lactate level. In patients with severe COPD lactate accumulation leads to the generation of $\mathrm{CO}_{2}$ and this, in turn, is thought to contribute to exercise cessation. The application of noninvasive pressure support (IPS) during exercise reduces dyspnoea, extends walking time, ${ }^{2}$ and unloads the inspiratory muscles. ${ }^{3}$ We therefore hypothesised that application of IPS during high intensity treadmill exercise in patients with severe COPD might prolong the duration of exercise induced lactataemia; if so, IPS might be a useful adjunct in pulmonary rehabilitation programmes for patients with severe COPD.
The subjects were eight men with severe stable COPD who had previously participated in exercise studies. The protocol was approved by our ethics committee and all subjects gave their written informed consent.

PROTOCOL

The patients performed two treadmill walks, separated by an interval of at least 30 minutes, at equal speed until they reached a condition of severe dyspnoea. The treadmill speed was determined in walks performed prior to the study day to be the subject's habitual brisk walk. The first walk was performed without IPS, during which subjects wore a tightly fitting face mask with a non-rebreathing two way valve attached (Hans Rudolph 2600 series, Kansas City Missouri, USA). During the second walk IPS was provided by a NIPPY ventilator (Air Med, London, UK) via a tightly fitting oronasal face mask. The ventilator settings were individually chosen prior to the walk to maximise patient comfort; typically the trigger was as sensitive as possible $\left(\mathrm{P}_{\text {mask }}=\right.$ $-0.5 \mathrm{~cm} \mathrm{H}_{2} \mathrm{O}$ ) with an expiratory time or two seconds and an inspiratory time of 0.8 seconds. The subjects used the ventilator for a few minutes while standing stationary on the treadmill before the walk, while the investigators made small adjustments to the settings to optimise patient comfort, and further adjustments were permitted during the walk.

\section{MEASUREMENTS}

During the free walk expiratory flow was obtained via a pneumotachograph placed in the expiratory limb of the non-rebreathing valve (Mercury Electronics CS5, Kilwinning, Scotland, UK) and integrated to give minute ventilation. We chose not to measure ventilation during the assisted walk because such a measurement would be difficult to interpret given that the ventilator compensates for mask leaks by delivering extra air.

Arterialised blood was drawn from a hand vein maintained at $42^{\circ} \mathrm{C}$ in a water bath. Samples were obtained at baseline and at the end of exercise for both the free and IPS assisted walks. An additional sample was drawn during the IPS walk at the time when the free walk finished ("isotime"). These samples were analysed for lactate using a YSI 2300 lactate analyser. A portion of each sample was also used for gas analysis.

ANALYSIS OF DATA

Statistics were computed using Wilcoxon's signed rank test (Statview 4.0, Abacus Con- 
Table 1 Mean (SD) exercise data

\begin{tabular}{llll}
\hline & Baseline & Isotime & End exercise \\
\hline $\begin{array}{l}\text { Free walk } \\
\mathrm{pH}\end{array}$ & $7.41(0.01)$ & & \\
$\mathrm{PCO}_{2}(\mathrm{kPa})$ & $5.38(0.42)$ & & $7.36(0.04) \# \ddagger$ \\
$\mathrm{PO}_{2}(\mathrm{kPa})$ & $7.54(1.26)$ & & $6.32(0.77) \# \ddagger$ \\
$\mathrm{Lactate}(\mathrm{mmol} / \mathrm{l})$ & $1.65(0.40)$ & & $2.59(0.87)$ \\
IPS assisted walk & & & \\
$\mathrm{pH}$ & $7.44(0.02) \dagger$ & $7.39(0.02)$ & $7.37(0.02) \#$ \\
$\mathrm{PCO}_{2}(\mathrm{kPa})$ & $4.99(0.57) \dagger$ & $5.83(0.60)$ & $5.86(0.67) \# \dagger$ \\
$\mathrm{PO}_{2}(\mathrm{kPa})$ & $8.47(1.19)$ & $6.16(1.13)$ & $5.84(1.27)$ \\
$\mathrm{Lactate}^{\star}(\mathrm{mmol} / \mathrm{l})$ & $1.53(0.44)$ & $2.42(0.79)^{\star}$ & $2.42(1.01)^{\star}$ \\
\hline
\end{tabular}

†Significantly different from free walk $(\mathrm{p}<0.05)$.

$\star$ Significantly different from pre-walk $(p<0.01)$.

- Significantly different from free walk $(p<0.01)$.

\#Significantly different from pre-walk $(\mathrm{p}<0.02)$.

$\ddagger$ Significantly different from IPS walk at isotime $(\mathrm{p}<0.04)$.

cepts, Berkeley, California, USA), a level of $\mathrm{p}<0.05$ being taken as significant.

\section{Results}

The subjects had a mean (SD) age of 70 (8) years with mean (SD) forced expiratory volume in one second $\left(\mathrm{FEV}_{1}\right)$ of $0.68(0.17)$ litres (24 (7) $\%$ of predicted).

In the free walk the subjects achieved a mean peak ventilation of $28 \mathrm{l} / \mathrm{min}$ (range 21-33). Mean (SD) walking time in the unassisted walk was 5.5 (1.5) minutes and in the assisted walk was 13.6 (6.0) minutes, an average increase of $149 \%(\mathrm{p}=0.01)$.

Mean exercise data are presented in table 1. Before exercise IPS resulted in a nonsignificant increase in $\mathrm{pH}$ and arterial oxygen tension $\left(\mathrm{PO}_{2}\right)$ and a fall in arterial carbon dioxide tension $\left(\mathrm{PCO}_{2}\right) \quad(\mathrm{p}<0.05)$. Free and IPS assisted walking were associated with a fall in $\mathrm{pH}(\mathrm{p}<0.02$ for both walks $)$ and $\mathrm{Po}_{2}(\mathrm{p}<0.02$ for both walks) and a rise in $\mathrm{PCO}_{2}(\mathrm{p}<0.02$ for both walks). Mean $\mathrm{pH}$ and $\mathrm{PO}_{2}$ at the end of exercise were not significantly different between IPS and free walks, but mean $\mathrm{PCO}_{2}$ was higher at the end of the free walk than the IPS walk $(p<0.04)$. Comparison of the data obtained at isotime showed significant differences in favour of IPS assisted walking for $\mathrm{pH}$ $(\mathrm{p}<0.04)$ and $\mathrm{PCO}_{2}(\mathrm{p}<0.04)$ but not for $\mathrm{PO}_{2}(\mathrm{p}$ $=$ NS).

Both free and IPS walks produced a significant $(p=0.01)$ rise in plasma lactate levels, the mean peak lactate level being $2.42 \mathrm{mmol} / \mathrm{l}$ at the end of the IPS walk and $2.96 \mathrm{mmol} / 1$ at the end of the free walk; the mean peak lactate level was significantly higher at the end of the free walk than at the end of the IPS assisted walk $(\mathrm{p}=0.01)$.

\section{Discussion}

Our data confirm that the use of IPS during treadmill exercise prolongs the duration of exercise and show that, as a consequence, IPS prolongs the duration of exercise induced lactataemia; this observation may be of value for the pulmonary rehabilitation of patients with severe COPD.

This study was not designed to test the hypothesis previously shown by us ${ }^{2}$ and others ${ }^{4}$ that IPS prolongs exercise duration in patients with COPD but rather that, in doing so, the lactataemia is prolonged. Nevertheless, our conclusions are only valid if the patients make maximal efforts during both walks. Our patients were experienced participants in physiological studies and the data from the free walk (mean VE $28 \mathrm{l} / \mathrm{min}$ and mean fall in $\mathrm{pH}$ of 0.05) are also consistent with a maximal effort. It is acknowledged that the magnitude of the observed increase in exercise duration may not be achievable in a less highly selected population.

The rise in lactate levels observed during the IPS walk was slightly less than that observed during free walking. One explanation for this could be that, in patients with COPD performing high intensity exercise, the respiratory muscles themselves make a small contribution to lactate production. In subjects with severe COPD Cooke et al failed to find a rise in lactate levels from hyperventilation alone, ${ }^{5}$ which suggests that the respiratory muscles do not significantly contribute to lactate production. However, Engelen et al compared voluntary hyperpnoea and whole body exercise in patients with moderate to severe $\mathrm{COPD}^{6}$ and found, like Cooke et al, that the origin of the lactate was principally non-respiratory but they considered that, in severely obstructed patients, hyperventilation alone could increase the lactate concentration by $0.5 \mathrm{mmol} / \mathrm{l}$. We therefore believe that the difference observed in peak lactate levels between free and IPS assisted walking could in part be attributed to unloading of the respiratory muscles resulting in a reduction in lactate generation.

The value of adjusting the exercise prescription to produce a lactataemia has recently been questioned by further data from Casaburi et al which showed that an appreciable benefit could be obtained in some severely obstructed patients who were unable to increase blood lactate levels during exercise. This improvement was obtained by the adoption of a more efficient breathing pattern such that respiratory rate decreased and tidal volume increased. However, we suggest that the demonstration of this mechanism does not preclude an additional physiological benefit from an exercise prescription which induces lactataemia as demonstrated by this group's previous study of patients with moderate COPD. ${ }^{1}$

The current data serve to generate a hypothesis that inspiratory pressure support used during exercise might increase the training benefit of pulmonary rehabilitation. This hypothesis is now being tested by a number of research groups, including our own; if it proves correct, further work will be required. In particular, the current data do not define the optimal prescription of IPS during exercisefor example, it remains to be determined whether IPS might be better employed to prolong the duration of exercise induced lactataemia or to heighten its intensity.

We conclude that IPS prolongs the duration of exercise induced lactataemia associated with steady state treadmill exercise in severe COPD. It is probable that IPS could facilitate physiologically useful pulmonary rehabilitation. Further clinical studies are warranted to address this. 
Funding: PH was supported by grant No. F97/1 from the National Lotteries Board administered by the British Lung Foundation. SGE was supported by a project grant from NHS Research \& Development (South Thames).

1 Casaburi R, Patessio A, Ioli F, et al. Reductions in exercise lactic acidosis and ventilation as a result of exercise training in patients with obstructive lung disease. Am Rev Respir Dis 1991;143:9-18.

2 Keilty SEJ, Ponte J, Flemming TA, et al. Effect of inspiratory pressure support on exercise tolerance and breathlessness in patients with severe stable chronic obstructive pulmonary disease. Thorax 1994;49:990-4

3 Polkey MI, Kyroussis D, Mills GH, et al. Inspiratory pressure support reduces slowing of inspiratory muscle relaxation rate during exhaustive treadmill walking in severe COPD. $A m \mathcal{F}$ Respir Crit Care Med 1996;154:1146-50.

4 Bianchi L, Foglio K, Pagani M, et al. Effects of proportional assist ventilation on exercise tolerance in COPD patients with chronic hypercapnia. Eur Respir f 1998;11:422-7.

5 Cooke NT, Wilson SH, Freedman S. Blood lactate and respiratory muscle fatigue in patients with chronic airways obstruction. Thorax 1983;38:184-7.

6 Engelen MPJK, Casaburi R, Rucker R, et al. Contribution of the respiratory muscles to the lactic acidosis of heavy exerthe respiratory muscles to the lactic acidosi
cise in COPD. Chest 1995; 108:1246-51.

7 Casaburi R, Porszasz J, Burns MR, et al. Physiologic benefits of exercise training in rehabilitation of patients with severe chronic obstructive pulmonary disease. Am $\mathcal{f}$ Respir Crit Care Med 1997;155:1541-51. 